\title{
GLAD!
}

Revue sur le langage, le genre, les sexualités

11 | 2021

Archives, genre, sexualités, discours

\section{« Débarrasser la société de femme[s] de ce genre- là ». Appréhender les archives judiciaires au prisme du genre pour enquêter sur les féminicides}

"Ridding society of wom[e]n of that kind": Using a Gender Perspective to Investigate Femicides in Court Records

\section{Margot Giacinti}

\section{OpenEdition}

Journals

Édition électronique

URL : https://journals.openedition.org/glad/3217

ISSN : 2551-0819

Éditeur

Association GSL

Référence électronique

Margot Giacinti, « « Débarrasser la société de femme[s] de ce genre-là ». Appréhender les archives judiciaires au prisme du genre pour enquêter sur les féminicides », GLAD! [En ligne], 11 | 2021, mis en ligne le 20 décembre 2021, consulté le 29 janvier 2022. URL : http://journals.openedition.org/glad/ 3217

Ce document a été généré automatiquement le 29 janvier 2022.

\section{(c) (1) $\Theta \Theta$}

La revue GLAD! est mise à disposition selon les termes de la Licence Creative Commons Attribution Pas d'Utilisation Commerciale - Pas de Modification 4.0 International. 


\title{
« Débarrasser la société de femme[s] de ce genre-là ». Appréhender les archives judiciaires au prisme du genre pour enquêter sur les féminicides
}

\author{
"Ridding society of wom[e]n of that kind": Using a Gender Perspective to \\ Investigate Femicides in Court Records
}

\section{Margot Giacinti}

Depuis les années 2000, par le biais de mobilisations collectives, particulièrement en Amérique Latine (Lapalus 2017; Segato 2016; Devineau 2012) et en Europe (Bodiou \& Chauvaud 2019; Cacouault-Bitaud \& Jaspard 2020), on a pu observer une diffusion rapide du terme "féminicide» dans l'espace public. Toutefois, en France, cette diffusion tant à l'échelle militante ${ }^{1}$ qu'à l'échelle institutionnelle ${ }^{2}$ s'est souvent effectuée au prix d'une définition limitée du fait social. En effet, la définition qui tend à s'imposer aujourd'hui ${ }^{3}$ se limite au cadre conjugal, c'est-à-dire au meurtre d'une épouse (ou concubine) par son compagnon ou son ex-compagnon. Le mot « féminicide», dans son acception française ${ }^{4}$, désignerait alors le seul féminicide conjugal. Toutefois, le concept de féminicide, développé par Jill Radford et Diana Russell (1992), avait vocation à décrire, dénoncer et politiser un phénomène global, varié dans ses manifestations et touchant la classe des femmes dans son ensemble, et non le seul meurtre d'une conjointe ou d'une épouse. Historiquement, il a été en général difficile pour les luttes féministes de faire accepter l'existence de violences à l'intérieur de la sphère conjugale (Hanmer 1977; Debauche \& al. 2013). Ce long combat des militantes les a notamment amenées à créer de toutes pièces des lieux d'accueil pour les femmes victimes de violences conjugales afin de pallier le manque de prise en charge de celles-ci, à l'instar de la création des premiers refuges pour femmes battues à la fin des années 1970 (Delage 2015). De plus, comme le rappellent Dominique Fougeyrollas-Schwebel et 
Maryse Jaspard (2003), il faut attendre les années 1990 pour que, sous la pression des militantes féministes, des campagnes institutionnelles de grande envergure sur les violences conjugales soient amorcées et qu'une réflexion sur un changement législatif en la matière débute, lequel entrainera entre autres conséquences plusieurs modifications du droit (par exemple l'aggravation de la pénalisation des violences conjugales en 1994).

2 Pourtant, dans le cas du féminicide, c'est l'inverse qui semble s'être produit, puisque celui-ci est désormais presque uniquement abordé en France par les institutions et les militantes comme un phénomène limité à la sphère du couple. Ainsi, si le contexte intime, voire familial, est généralement négligé par les institutions en matière de violences exercées contre les femmes, pour la question qui nous occupe, il est souvent le seul cadre de référence accepté par les acteurs politiques et militants. Plusieurs hypothèses peuvent être avancées pour tenter d'expliquer ce phénomène. Sur le plan légal et politique d'abord, les travaux ont montré de quelle manière l'arsenal judiciaire et législatif français, notamment à travers les Codes napoléoniens (Code civil de 1804, Code d'instruction criminelle de 1808, Code pénal de 1810), a véhiculé une idéologie conjugaliste et familialiste (Verjus 2010) dont a hérité l'appareil judiciaire contemporain ${ }^{5}$. En effet, le phénomène social du féminicide a été historiquement saisi au prisme du couple, comme en témoignent les débats sur la création de la catégorie de conjugicide dans le Code pénal de 1810 (Vanneau 2016: 63-66) ou encore les prises de position en faveur du rétablissement du divorce pour réduire la fréquence des meurtres entre époux (Giacinti 2020: 55-56). De plus, bien que le mot féminicide existât en français au XIX siècle dans des corpus divers (écrits journalistiques, politiques, juridiques, cf. Giacinti 2020), son utilisation pour renvoyer à des formes variées, mais comparables, de violence meurtrière à l'égard des femmes reste trop marginale pour parler d'une véritable conceptualisation. Par ailleurs, cette fois sur le plan de l'analyse sociohistorique des archives judiciaires en France, les études se sont souvent intéressées aux homicides conjugaux davantage qu'aux féminicides en général (Gagnon 2002 ; Crépin 2016). Enfin, sur le plan discursif, Silvia Nugara a pu montrer l'influence $\mathrm{du}$ discours des institutions internationales sur l'émergence d'une définition réduite du féminicide en Europe (2011). En effet, si le cas des meurtres massifs de femmes en Amérique Latine, particulièrement au Mexique, a conduit les institutions locales à admettre l'existence d'un fait social massif, les observateur-trice's européen-ne's se sont révélée's réticente-s à accepter l'existence d'un féminicide au sens de "meurtre de femmes de masse » sur ce territoire, entrainant une définition du féminicide restreinte à la seule sphère conjugale (ibid. : 180-181). De plus, bien que les enquêtes Enveff (2000) et Virage (2015-2020) aient documenté le fait que les femmes subissent des violences dans d'autres sphères que le seul cadre conjugal, la lecture conjugaliste du féminicide persiste en France 6 . Prenant appui sur la définition de Diana Russell du féminicide comme le meurtre d'une femme "parce qu'elle est une femme » (2001, nous soulignons), nous aimerions souligner l'intérêt heuristique d'une telle définition en développant deux conditions d'identification du féminicide à partir de l'expression "parce qu'elle est une femme ». Non seulement le féminicide est un crime dans lequel la victime est une femme ou une personne perçue comme telle mais, de plus, le féminicide est un crime dans lequel le genre est déterminant dans les circonstances du meurtre. À partir de ces deux conditions, il est possible de travailler en féministe ${ }^{7}$ la sélection des cas de féminicides dans les archives judiciaires et leur étude, afin de s'interroger sur ce qui a 
pu faire écran à une lecture unifiée d'un phénomène pourtant matériellement présent dans les sources ${ }^{8}$.

Dans un premier temps, nous présenterons le matériau d'archive utilisé. Puis, nous analyserons par le prisme du genre les catégories théoriques adoptées par les acteurs prenant part à l'enquête policière et à la procédure judiciaire, ainsi que leurs pratiques d'investigation. Les catégories utilisées pour observer ces meurtres sont celles « universelles» du droit pénal: homicide volontaire, involontaire, motivé, précédé, accompagné ou suivi de vol, etc. Elles ne permettent donc pas de saisir dans ces cas divers un fait social structurel. Les pratiques de ces acteurs - qui appartiennent à un appareil judiciaire exclusivement composé d'hommes (Farge 1997) - témoignent d'une lecture biaisée des rapports sociaux.

Dans un second temps, nous montrerons comment une perspective féministe sur ces archives permet de modifier le regard porté sur les motivations de ces crimes. Malgré leur diversité, ces dossiers font apparaitre des logiques similaires qui poussent les hommes à tuer les femmes: exercice de la domination masculine, refus de l'émancipation, exploitation de la vulnérabilité structurelle des femmes. L'analyse des archives par le genre rend alors possible de subsumer ces faits sociaux divers sous la catégorie unitaire de féminicide.

\section{Enquêter sur les féminicides dans les archives judiciaires du XIXe siècle}

5 Enquêter sur les féminicides dans les archives du XIX ${ }^{e}$ siècle ${ }^{9}$ exige, comme l'affirme Liora Israël, d'« inventer ses archives»(2012: 177). En effet, si les dossiers de procédure des procès jugés par la cour d'assises du Rhône se trouvent essentiellement regroupés dans la série U (Justice, Cour d'Assises) des archives départementales du Rhône, la catégorie de féminicide n'existe pas en droit français. Enquêter sur les féminicides nécessite alors de contourner cette absence par l'usage d'archives dans lesquelles le chef d'accusation retenu est l'homicide, en sélectionnant les homicides dans lesquels la victime est une femme ${ }^{10}$. Les dossiers de féminicide relèvent alors de catégories pénales diverses. Les plus évidentes sont celles de l'homicide prémédité (assassinat) ou de l'homicide non prémédité (meurtre ${ }^{11}$ ). À ces deux premières catégories, on peut ajouter le parricide ${ }^{12}$, l'infanticide ${ }^{13}$, ou encore l'empoisonnement ${ }^{14}$. Il faut encore adjoindre à ces catégories pénalement définies les tentatives de crime ${ }^{15}$. Les tentatives d'assassinat, de meurtre, de parricide, d'infanticide ou d'empoisonnement sont jugées par le droit comme étant assimilables au crime. Parce qu'ils présentent généralement les témoignages des victimes, les dossiers de procédures de tentatives de féminicides sont intéressants pour notre étude : ils permettent en effet de saisir directement la parole des victimes, ce qui n'est habituellement pas possible dans les féminicides effectifs ${ }^{16}$. Enfin, les dossiers peuvent aussi relever de la catégorie des «coups et blessures mortels", introduite par la loi du 28 avril $1832^{17}$. Visant à assouplir les sanctions associées au crime, cette réforme est présentée comme un moyen d'éviter que les jurys acquittent les accusés dont l'enquête a pourtant établi la culpabilitée ${ }^{18}$, et ce, par crainte qu'ils soient condamnés à la peine capitale. Est ainsi ajoutée dans l'article 309 du Code la situation dans laquelle les coups donnés occasionnent la mort ${ }^{19}$. En l'absence d'une catégorie pénale définissant le féminicide, le corpus d'archives étudié est donc constitué de dossiers dont le chef d'accusation relève de différentes 
catégories du droit. Nous excluons de la présente étude le cas des infanticides, qui, au regard de la typologie exposée, peuvent pourtant entrer dans la catégorie des féminicides. En effet, deux problèmes principaux se posent au sujet de la recherche des féminicides parmi les infanticides, l'un relatif au contenu des sources et l'autre relatif à des questionnements épistémologiques. D'une part, les archives d'infanticides sont très nombreuses dans les archives judiciaires (Tillier 2001) et ne mentionnent pas toujours le sexe de la victime. D'autre part, étant donnée la criminalisation de l'avortement sur la période étudiée et en l'absence de méthodes contraceptives et abortives efficaces, l'infanticide comme fait social peut s'apparenter à des pratiques d'avortement dont la logique différerait alors de celle des féminicides ${ }^{20}$ : l'infanticide est utilisé pour se débarrasser de tout enfant, qu'il soit un garçon ou une fille et ne vise pas spécifiquement l'élimination des petites filles, à l'inverse des néonaticides sexosélectifs (Giacinti 2020).

\section{Les archives de féminicides, témoins de pratiques policières et judiciaires genrées}

Reconstituer les faits a posteriori n'est jamais commode, d'autant que la plupart des dossiers offrent in fine une version qui est bien souvent celle de l'ordre public et des autorités de police. Les questions posées ont l'évidence des certitudes policières: avant tout, l'homme de police cherche à nommer des coupables, peu lui importe que l'affaire soit totalement éclairée. (Farge 1997 : 106)

Cette réflexion d'Arlette Farge nous permet de nous intéresser plus spécifiquement au contenu des archives, et en particulier aux pratiques policières et judiciaires comme à des processus qui co-construisent le féminicide comme fait social. Les dossiers de procédures étudiés, issus de la période 1860-1890, sont constitués des pièces de l'information judiciaire, c'est-à-dire de l'ensemble des pièces que les autorités policières et judiciaires produisent pour éclairer les circonstances dans lesquelles le crime s'est déroulé, qualifier les faits et trouver le(s) coupable(s). On y trouve aussi bien le procès-verbal de transport établi par la police judiciaire, qui décrit la découverte du corps de la victime, interroge les premiers témoins et dresse les premières observations, que le document qui récapitule les questions posées au jury de la cour d'assises du Rhône ${ }^{21}$. Dans ce processus, un des objectifs de l'enquête est de qualifier l'acte : le crime est-il un meurtre? Un assassinat? Fait-il suite à des coups mortels sans intention de donner la mort? Ces questions sont importantes car la réponse est indispensable à la bonne tenue de l'enquête. En effet, la qualification du crime est nécessaire à l'établissement de l'acte d'accusation, produit par le procureur général, qui détermine les questions posées au jury de la cour d'assises, et donc la sanction.

\section{Les récits stéréotypés des auteurs de féminicides}

7 Pour qualifier le crime, il faut déterminer l'intention de l'auteur, ce qui conduit les policiers et les juges à interroger tour à tour les témoins du crime et l'accusé lui-même. Dans certains cas, l'accusé avoue parfois le crime dans son ensemble, sans cacher son intention, ce qui a pour conséquence un traitement rapide de l'enquête et donc une production peu importante de pièces judiciaires ${ }^{22}$. Mais ces situations restent relativement rares, et dans la majorité des cas, l'accusé produit une défense, seul ou accompagné de son avocat, et propose un récit de l'évènement visant à atténuer la sanction, en tentant par exemple d'amoindrir sa responsabilité dans le crime. Dans les 
cas de féminicides, la défense de l'accusé prend appui de manière récurrente sur des représentations communes, qui révèlent des biais de genre et renseignent sur les rapports sociaux de sexe. On peut dégager ici à titre d'illustration quatre récits masculins typiques visant à justifier le féminicide (ou sa tentative ${ }^{23}$ ) :

- L'accusé affirme avoir été trompé par la victime, comme c'est le cas dans l'affaire Laurent (1880) dans laquelle l'accusé, qui a jeté du vitriol sur sa femme, s'en défend ainsi : « Mon seul but était de la défigurer afin de l'obliger à changer de conduite. Ma femme m'a dit à moimême qu'elle avait eu des relations avec le sieur Barthelemy [...], son père, employé de commerce $[. . .]^{24}$.

- L'accusé allègue que la victime voulait le quitter ou l'avait quitté, comme c'est le cas dans l'affaire Duvivier (1878). Dans celle-ci, l'accusé tente de donner la mort par balle à son ancienne maitresse qui l'a quitté. Dans un message, présenté comme un testament et destiné à la police, il écrit : «Elle ajouta que je n'avais aucun droit sur elle et qu'elle ne m'avait pas promis fidélité en me quittant ; oh que j'aurais déjà voulu avoir l'arme dont je me suis servi aujourd'hui' ${ }^{25}$."

- L'accusé met en avant le refus de la victime de l'épouser, comme c'est le cas dans l'affaire Talagrand (1876), dans laquelle l'accusé justifie sa tentative de féminicide contre sa victime ainsi : «Mon intention était bien arrêtée de la tuer et moi après. La haine que j'ai contre elle résulte de ce qu'elle m'a refusé formellement le mariage avec moi et qu'elle parle à un autre jeune homme ${ }^{26}$."

-Enfin, l'accusé avance que sa victime ne remplissait pas bien le rôle qui était attendu d'elle. Dans l'affaire Bonnin (1883), l'accusé est l'époux de la victime et justifie la tentative d'assassinat par le refus d'avoir des relations sexuelles que lui oppose son épouse. Lors d'un des interrogatoires menés par le juge d'instruction, il indique ainsi : « Ma femme me rendait malheureux. Je lui apportais bien l'argent que je gagnais et cependant voilà cinq mois qu'elle ne couche pas avec moi $^{27}$. »

8 Ces quelques exemples donnent un aperçu des défenses genrées produites par les accusés pour tenter d'expliquer leur crime. Elles s'appuient en effet sur des stéréotypes de genre (infidélité de la victime, refus injustifié de sa part, manquement à ses « devoirs » conjugaux, etc.) et des pratiques discursives qui visent à rendre les victimes responsables de leur propre mort. Ces logiques, qui se combinent, tendent à faire accepter à l'interlocuteur la légitimité d'agir pour se faire justice ou pour sauver son honneur. Mais ces récits masculins, loin de rester de simples paroles à laquelle l'institution accorderait peu d'importance, sont saisies par les policiers et les juges. Ces derniers, dans la logique de l'enquête judiciaire, interrogent la véracité des propos de l'accusé, et évaluent par ce même processus la crédibilité qui peut lui être accordée. Lorsque la version de l'accusé trouve grâce aux yeux de l'institution, soit parce qu'elle est vérifiée lors de l'information soit parce qu'elle parait légitime, il peut attendre une plus grande bienveillance, par exemple par l'admission de circonstances atténuantes ${ }^{28}$. Par ailleurs, la légitimité de la version de l'accusé repose sur un système de représentations dans lequel les rôles sociaux attendus des hommes et des femmes sont déterminés par leur genre. Ainsi, lorsque les policiers enquêtent sur un féminicide et cherchent à " élucider le monde social », pour reprendre les mots de Dominique Kalifa (2010: 6), ils peuvent être amenés à reconstruire les faits à partir de la version de l'accusé, notamment dans les cas où la victime a été tuée ou ne peut témoigner. À toutes les étapes de l'enquête, cette reconstruction peut alors avoir lieu sur des bases partielles et genrées, défavorables à la victime, comme il s'agit de le montrer à présent. 


\title{
Recherche du motif du crime : une pratique défavorable aux victimes de
} féminicides?

La recherche du ou des motif(s) du crime est une partie importante de l'enquête judiciaire. Elle vise à établir les raisons qui ont amené l'accusé à commettre le crime. Dans la logique policière, la recherche du motif passe souvent par l'interrogatoire de l'accusé et par l'audition de la victime, des témoins et des experts. Cependant, cette partie de l'enquête n'interroge que peu les rapports de genre et participe à construire des solidarités masculines défavorables à la victime. Ainsi, bien que l'institution judiciaire puisse avoir des doutes sérieux quant à la véracité du propos de l'accusé, elle peut être amenée à reprendre le motif allégué et à le faire figurer tel quel dans l'acte d'accusation, pièce centrale puisqu'elle dresse les circonstances dans lesquelles le crime a eu lieu et permet de qualifier pénalement les faits. Parce que le motif renvoie souvent à un système de représentations genrées commun de l'époque, il peut alors figurer dans l'acte d'accusation, même lorsque l'enquête a démontré son inexactitude. Dans l'affaire Hervier (1881), l'acte d'accusation reprend le motif indiqué par l'accusé (la jalousie) tout en remettant en question le fondement de ce motif : "D'un autre côté, Hervier était sans aucune espèce de motifs d'une jalousie extrême et entrait dans de grandes colères au sujet de la prétendue infidélité de sa femme ${ }^{29}$. $"$ Le document rend également compte des propos de l'accusé au moment de son arrestation : "L'accusé n'a pas essayé de nier le crime qu'il a commis, mais il a prétendu qu'il avait été poussé par le refus de sa femme d'accomplir le devoir conjugal ${ }^{30}$. " Pourtant, l'enquête avait bien émis des doutes sur la véracité du motif donné par l'accusé et avait établi que ce dernier avait contracté de nombreuses dettes, ce qui donnait lieu à des reproches de la part de sa femme. Dans l'acte d'accusation cohabitent ainsi le motif établi par l'enquête et le motif donné par l'accusé, qui se retrouve ainsi légitimé par sa seule présence dans le document. Parfois, lorsque la recherche du motif n'est pas suffisamment concluante pour la justice, la justice peut alors orienter l'enquête. Dans l'affaire Vermorel (1878), dans laquelle l'accusé a tué son épouse à coups de hache, l'institution judiciaire piétine. Elle demande alors à ce qu'un médecin procède à un examen mental de l'accusé :

\begin{abstract}
Attendu que ni l'instruction ni les débats n'ont pu établir d'une façon suffisante l'existence d'un motif qui aurait pu déterminer Vermorel à accomplir l'acte qui lui est reproché, que Vermorel lui-même ne peut donner aucune explication raisonnable de sa conduite, que Vermorel n'avait aucun intérêt d'une nature quelconque à la mort de sa femme, attendu qu'en présence d'un acte aussi grave commis sans motif appréciable par un homme jusque-là honnête, il y a lieu de se demander si l'accusé n'a pas agi sous l'influence d'un accès de folie. Que cette possibilité doit être l'objet d'un examen d'autant plus sérieux qu'il y a eu dans la famille tant paternelle que maternelle de l'accusé des cas constatés de folie et d'épilepsie et qu'aussi le caractère sombre et taciturne de l'accusé était de ceux qu'on remarque chez les personnes prédisposées à l'aliénation mentale ${ }^{31}$.
\end{abstract}

10 Dans cette affaire, la violence masculine comme cause du féminicide n'est pas envisagée comme un motif propre, ce qui amène la justice à s'interroger sur l'état mental de l'accusé, et ce, bien que les renseignements établissent que Vermorel était un homme " violent, ivrogne, [...] sournois $»^{32}$. La recherche du motif du crime peut donc véhiculer des questionnements stéréotypés, qui ne prennent pas en compte la violence des hommes sur les femmes, et réhabilitent et légitiment des motifs sexistes du crime, alors 
même que l'enquête révèle que ces motifs sont inexacts. En somme, la présence même de la version de l'accusé rend perceptible une vérité policière et judiciaire.

\section{Quelle version retenir ? Quand la réputation de la victime prend le dessus sur les faits}

11 L'enquête judiciaire peut également rencontrer des difficultés dans l'établissement des faits. Dans l'affaire Mercier (1880), l'accusé a défenestré sa victime, une «fille soumise ${ }^{33}$ » qui décède quelque temps plus tard à l'hôpital sans avoir pu être entendue par les policiers. Avant de mourir, celle-ci a tout de même pu parler au chirurgien de son service, dont le témoignage figure dans l'examen médical : « Elle finit par m'avouer qu'elle avait été battue et assommée par un individu auquel elle avait refusé de se livrer après avoir été emmenée par lui, et cela parce qu'elle avait $b^{34}$.» Lors de son interrogatoire, l'accusé, à l'inverse, affirme que c'est la victime, ivre, qui voulait coucher avec lui, mais qu'il s'y était refusé et que sa chute n'était qu'un accident: "J'affirme que je n'ai pas jeté cette femme par-dessus la rampe de l'escalier. Si elle est tombée, elle est tombée en descendant l'escalier parce qu'elle était en état d'ivresse. Quant à moi je suis étranger aux conséquences de cette chute ${ }^{35}$.» L'enquête démontre que la chute ne pouvait pas être accidentelle, mais échoue à comprendre précisément le déroulement des faits. Toutefois, l'acte d'accusation donne une place importante à la version de l'accusé, alors qu'elle résume rapidement celle de la victime :

Transporté à l'Hôtel Dieu, la fille Vergoin y succomba le 17 juillet après avoir déclaré à plusieurs personnes que Mercier l'avait battue puis jetée par la fenêtre. Interrogé par M. le Commissaire de police le 19 juillet, l'accusé niait avec énergie avoir amené chez lui la fille Vergoin. Plus tard devant M. le juge d'instruction, il dut reconnaitre que sa première déclaration était mensongère. Il avoua alors que la fille Vergoin était venue dans son appartement conduite par lui. Elle était ivre et faisait du bruit. N'ayant pu la calmer il l'avait mise à la porte et il ignorait ce qu'elle était devenue depuis, si elle était tombée dans la cour c'était suivant l'accusé, le résultat d'un pur accident $^{36}$.

12 Cet extrait met en évidence qu'en plus d'être rapporté avec plus de détails, le discours de l'auteur du crime est repris sans effet de citation, alors même qu'il reproduit strictement les mots de l'accusé. Cette récupération est révélatrice du fait que cette version prend le dessus sur celle de la victime. Par ailleurs, bien que l'acte établisse que la défense de l'accusé soit « inadmissible et se trouve démenti[e] par les constatations qui ont été faites et par les témoignages que l'information a recueillis ${ }^{37}$ », il tend à valider la version de l'accusé en suggérant que ce n'est pas parce que sa victime refusait de se livrer à lui que l'accusé l'a battue et jetée par la fenêtre, mais parce que sa présence et ses cris l'auraient compromis : «Il est donc certain que pour se débarrasser d'une fille dont la présence et les cris persistants étaient de nature à le compromettre, l'accusé n'a pas reculé devant un crime ${ }^{38}$. Tout aussi remarquable est le fait que la défense de l'accusé repose tout au long de cette enquête sur la mauvaise réputation de la victime, elle-même étroitement liée à sa situation de prostitution. Dans les interrogatoires, l'accusé rappelle que «cette fille voulait évidemment faire du scandale $^{39}$ ». Plus généralement, la victime est décrite par le médecin comme "une malheureuse ${ }^{40}$ ", menant « une vie dissolue ${ }^{41}$ », tandis que sa sœur déclare qu'elle « ne [la] fréquentai[t] pas, parce qu'elle menait une mauvaise vie ${ }^{42} »$. Si l'on prend en 
compte que Mercier n'est pas reconnu coupable de meurtre, mais seulement de coups mortels, et qu'il est puni d'une sanction très faible en comparaison des peines de la même période ${ }^{43}$, il est possible de formuler l'hypothèse que la réputation de la victime a eu ici un effet dans la qualification de l'acte. Les études portant sur le rôle joué par la réputation dans le jugement (Claverie 1979; Tanguy 2000 ; Ferron 2002 ; Vanneau 2016) révèlent l'importance des normes de genre dans l'enquête, qui peut jouer au bénéfice de l'accusé lorsque la victime n'est pas une «femme respectable». Dans le cas Mercier, le fait même que la victime soit une fille soumise semble bénéficier à l'accusé, et comme le rappellent d'autres affaires, la prostitution ou la suspicion de prostitution place les victimes en situation d'immoralité, donc de vulnérabilité structurelle, puisqu'elles ne sont pas jugées sur un pied d'égalité avec les autres citoyennes (Plumauzille 2016; Giacinti 2020). Mais la prostitution n'est pas la seule situation dans laquelle la victime risque d'être jugée immorale. En effet, cette dernière peut être perçue coupable de mauvaises mœurs dans le cas où elle a eu des relations sexuelles avec un homme hors d'une relation maritale, par exemple si ces relations ont eu lieu dans le cadre du concubinage. Dans l'acte d'accusation de l'affaire Duvivier (1878), la victime est présentée comme ayant « entretenu des relations coupables avec l'inculpé ${ }^{44}$ » et comme ayant même " pendant un certain temps vécu maritalement avec lui ${ }^{45}$ ", ce qui lui cause un discrédit certain. Toutefois, la question de la bonne moralité des femmes victimes de féminicides apparait surtout comme un prétexte pour renforcer un système de domination masculine. De fait, même des femmes à la réputation intacte peuvent être présentées comme responsables de leur sort. L'affaire Vermorel citée supra laisse ainsi apparaitre dans son acte d'accusation de vraies injonctions contradictoires qui reposent sur les femmes en général, et sur les victimes de féminicides en particulier :

Les voisins de Vermorel avaient au contraire remarqué la conduite irréprochable de sa femme. Laborieuse, constamment préoccupée de ses enfants et de son ménage, elle avait su s'attirer l'estime générale. Souvent, elle cherchait peut-être même avec trop de vivacité à combattre le penchant de son mari pour la boisson. Aussi s'en suivait fréquemment des discussions qui quelquefois dégénéraient en querelles sérieuses ${ }^{46}$.

$13 \mathrm{Au}$ lieu de s'en tenir à la mention de la bonne conduite de la victime, le procureur fait un lien entre son comportement, pourtant «irréprochable ", à savoir ses efforts pour que son mari boive moins, et son assassinat. Est ainsi suggérée l'idée qu'il aurait mieux valu, dans son propre intérêt, tolérer l'alcoolisme de son époux plutôt que de se montrer trop vertueuse en le combattant. L'injonction à la bonne moralité est ainsi retournée contre la victime, et l'on peut se demander si le véritable problème, dans le cas des femmes exerçant la prostitution comme dans celui de cette épouse vertueuse, ne réside pas dans leur position de résistance face à la domination masculine, qui se manifeste dans l'exercice de leur agentivité. Quelle qu'ait été l'attitude des victimes avant qu'elles ne soient tuées, leur posture est mécaniquement interrogée et commentée par des acteurs de la procédure judiciaire, ce qui a pour effet de rendre suspecte, voire compromettante, toute forme d'agentivité des femmes ${ }^{47}$.

14 Ainsi, les catégories du droit tout comme les pratiques des acteurs prenant part à l'enquête policière et à la procédure judiciaire révèlent une prise en compte minime des rapports sociaux de sexe dans l'analyse des féminicides. Par la recherche du motif du crime sans considérer les rapports de pouvoirs entre accusé et victime, en privilégiant la version de l'accusé au détriment de celle de la victime, en recherchant 
des renseignements sur la victime et en analysant sa moralité, notamment à partir de ses pratiques sexuelles, l'enquête échoue à saisir les rapports sociaux effectifs de sexe. Elle concourt à établir des procédures d'instruction dont le déroulé et la grille de lecture sont le produit d'une analyse exclusivement masculine. Par conséquent, sur le plan des catégories tout comme sur le plan des pratiques, s'entremêlent des logiques androcentrées qui font écran à la possibilité d'analyser le meurtre des femmes comme un fait social unitaire et structurel, c'est-à-dire comme des féminicides.

\section{Des logiques similaires à l'œuvre ? Le féminicide comme fait social unitaire}

Cette seconde partie interroge les données sociales elles-mêmes du féminicide en serrant au plus près les dénominateurs communs et les divergences. Pour ce faire, trois cas d'étude, les affaires Duvivier, Gonssolin et Grosselin, jugées en cour d'assises du Rhône entre 1878 et 1888 et issues des archives départementales du Rhône (AD69), seront mobilisés. Après avoir présenté brièvement ces affaires à partir des pièces produites par la justice, on montrera qu'une perspective féministe sur ces archives permet de saisir des logiques de genre similaires à l'œuvre dans ces crimes.

\section{Trois affaires a priori très différentes}

Ces trois affaires, deux féminicides et une tentative de féminicide, constituent a priori trois cas aux circonstances bien différentes, si l'on observe les liens entre la victime et l'accusé, les motifs établis par l'enquête ou encore le traitement judiciaire qui leur est réservé. L'affaire Duvivier (1878), tout d'abord, concerne Charles Duvivier, 37 ans, manœuvre, accusé d'avoir tenté de tuer Marie Portal, femme Moussy, coiffeuse, 41 ans. L'enquête établit que l'accusé et la victime avaient une relation depuis quelques années (1872), relation qui avait commencé alors que la seconde était mariée et que Charles Duvivier habitait dans la même maison. À la mort de son père, Charles Duvivier fréquentait les époux Moussy en venant notamment manger chez eux. C'est à ce moment-là que des relations se sont établies entre la victime et l'accusé, relations que tolérait le mari de la victime. Cette dernière explique qu'« en 1875, ennuyée de cette vie, [elle] voulu[t] en finir et [elle] se rendi[t] à Saint-Étienne chez [s]a sœur ${ }^{48}$ ", tout en continuant à fréquenter Duvivier à Lyon, qui « vint à [s]on secours dans ce moment-là et [lui] donna même de l'argent pour apprendre l'état de coiffeuse [qu'elle] exerce aujourd'hui ${ }^{49}{ }^{\prime}$. Cependant, la victime s'aperçoit rapidement que Duvivier ne travaille pas et qu'il profite de son propre salaire. À la date du 14 janvier, l'accusé se présente à Marie Portal, prétextant qu'il doit lui remettre une lettre importante. Sans se méfier, elle le suit et faisant le geste de lui remettre la lettre, il tire cinq coups de feu dont aucun ne blesse la victime, qui a eu le réflexe de se baisser et de fuir. Une balle est arrêtée par les vêtements de la victime. L'accusé fuit, et ce n'est que le 17 février qu'il est arrêté, après avoir tenté une seconde fois de tuer la victime: il rôde depuis la première tentative autour de son domicile et, dans la soirée du 16 février, piège Marie Portal, lui dit : "Cette fois tu ne m'échapperas pas ${ }^{50}$ ", et lui tire à nouveau dessus. Elle en réchappe une seconde fois. Dans un long testament retrouvé par la police, l'accusé explique les causes supposées de son crime (la jalousie), alléguant que sa victime l'avait quittée alors même qu'il l'avait aidée à devenir coiffeuse en payant sa formation. 
Charles Duvivier est condamné pour tentative d'assassinat, la préméditation ayant été établie, et condamné à quinze ans de travaux forcés.

Dans le second cas, celui de l'affaire Gonssolin (1880), Louis Gonssolin, 28 ans, anciennement garçon charcutier, tue sa maitresse Anna Lagros, « fille soumise » de 38 ans, dont il était aussi le souteneur et avec qui il habitait. L'enquête établit que Louis Gonssolin vivait de la prostitution d'Anna Lagros, qu'il maltraitait souvent. La victime s'était confiée à ses amies prostituées des violences qu'elle endurait; lorsqu'on lui conseillait de rompre, elle répondait: "J'en ai bien envie seulement ce qui me fait rester avec lui c'est la crainte, car je sais que si je le quitte, il me tuera ${ }^{51}$. » À plusieurs reprises, elle était allée au commissariat de police du quartier pour se plaindre qu'elle était maltraitée, y avait fait constater ses blessures et avait demandé de l'aide. Par ailleurs, Louis Gonssolin jouait beaucoup au jeu et avait, peu avant le meurtre, perdu de grosses sommes, se trouvant alors sans ressources financières. L'acte d'accusation indique qu'Anna Lagros, finalement résolue à quitter Louis Gonssolin, avait décidé de faire enlever sa malle de la chambre dans laquelle elle vivait avec lui. Ce dernier avait fait de même en envoyant une balle de linge à sa mère. Le lendemain, 8 mars, Louis Gonssolin tuait Anna Lagros par étranglement dans le logement commun, avant de donner lui-même l'alerte à la police en tentant de faire croire à un vol accompagné d'un meurtre et d'un viol. Les témoignages sur la brutalité de l'accusé sont nombreux et permettent à l'enquête d'établir la culpabilité de l'accusé malgré ses dénégations. L'examen médical des organes génitaux de la victime établissent que «la fille Lagros n'avait pas reçu le contact d'un homme ${ }^{52}$ ", tandis que l'enquête montre que "le désordre de la chambre paraissait simulé53 ${ }^{53}$. Présenté comme un homme de mauvaise réputation, déjà condamné une fois pour coups et blessures et une fois pour vol, Louis Gonssolin est condamné pour assassinat aux travaux forcés à perpétuité.

La troisième affaire étudiée est l'affaire Grosselin (1888), dans laquelle Marie Descroix veuve Claitte, âgée de 53 ans, est tuée et volée par Antoine Grosselin, 23 ans, sans profession, déjà condamné six fois pour vol et vagabondage. Marie Descroix est décrite comme "vivant dans une situation de fortune relativement aisé $\mathrm{e}^{54}$ ». Cette femme vivait seule avec son domestique et avait appris, peu avant son assassinat, la sortie de prison de Grosselin. Le 22 janvier 1888, le domestique de la veuve Claitte, qui " connaissa[i]t les habitudes très régulières de sa maitresse » fut surpris de trouver la porte du logement fermé et partit chercher la veuve Claitte dans les maisons du village, sans succès. En forçant la porte de la maison, les personnes du village constatent le désordre régnant dans le logement et trouvent Grosselin caché sous un lit, en possession d'un couteau taché de sang. Le cadavre égorgé de la Marie Descroix est retrouvé sous un tas de foin dans le fenil. L'enquête établit que Grosselin, qui " connaissait parfaitement les habitudes ${ }^{55}$ » de la victime, avait « attend[u] le moment favorable à l'exécution de ses projets ${ }^{56} »$. Il est condamné en premier lieu à la peine de mort pour assassinat commis avec guet-apens dans le but de préparer, faciliter ou exécuter un vol d'objets mobiliers sur la personne de Marie Descroix, et est, en plus, reconnu coupable d'avoir volé des objets mobiliers au domestique de la victime. Sa peine est finalement commuée en travaux forcés à perpétuité.

Ces trois affaires sont appréhendées par la justice comme : une tentative d'assassinat entre ex-amants, un assassinat d'un souteneur sur sa fille soumise, et un assassinat motivé par le vol ${ }^{57}$. Par ailleurs, on remarque de prime abord la forte hétérogénéité des cas. Qu'il s'agisse de l'âge des victimes (Marie Portal, 41 ans; Anna Lagros, 38 ans; 
Marie Descroix, 53 ans) de leur statut socio-économique (coiffeuse; fille soumise; rentière), de leur statut marital (séparée de son mari ; en relation avec son souteneur; veuve), les données ne semblent pas convergentes, et l'on aurait du mal, en première lecture, à y trouver trois variations du même fait social. Il est d'ailleurs probable que seul le premier cas, qui ressortit au féminicide dans la sphère conjugale, serait qualifié aujourd'hui de féminicide par les pouvoirs publics et une partie des militantes féministes françaises. Toutefois, un regard féministe sur ces trois cas permet de mettre en lumière des convergences, tout autant dans les profils sociologiques que dans le script du passage à l'acte. Derrière l'apparence disparate, on retrouve ainsi les caractéristiques communes d'un fait social unitaire.

\section{Enquêter en féministe dans les archives : diversité des féminicides, unité du fait social}

L'analyse de l'archive judiciaire comme seul et premier matériau, dit Arlette Farge, «suppose un choix et conduit un itinéraire» $(1997: 37)$. Si le fait de travailler sur la seule archive judiciaire est un choix, l'analyser en féministe en est un autre. Plus que jamais, ce positionnement doit prendre en compte que, dans l'archive judiciaire, «la parole retenue est contenue au cœur du système politique et policier [...] qui la gouverne et la produit ${ }^{58}$ » (ibid. : 39). En analysant ces crimes au prisme du genre, la chercheuse féministe peut envisager l'archive comme un témoin des rapports sociaux de sexe à l'œuvre entre femmes victimes et hommes, que ces hommes soient sur le banc des accusés ou qu'ils enquêtent. Ce positionnement permet d'interroger l'archive judiciaire sous des angles rarement saisis par les logiques policières ou institutionnelles, en tentant d'appréhender et de rétablir, par exemple, le récit et le vécu des victimes. Cette démarche s'inscrit dans une perspective féministe dans la mesure où elle organise l'enquête autour du genre comme catégorie d'analyse, ce qui permet d'interroger le biais de l'archive et des discours qu'elle contient, en travaillant à faire advenir les voix tues par la constitution androcentrée du matériau archivistique.

Dans les trois affaires, une lecture serrée du discours des victimes (lors de l'enquête ou avant d'être tuées), permet d'abord d'identifier un sentiment de crainte à l'égard de leur meurtrier. Que les victimes soient dans une relation d'ordre intime avec leur agresseur (affaires Duvivier et Gonsollin) ou non (affaire Grosselin), la victime a, directement ou indirectement, exprimé à l'institution policière et/ou à des proches la crainte que lui inspirait l'accusé. Celle-ci peut se manifester à l'égard de violences déjà subies et pouvant être renouvelées ou de violences larvées, que la répétition des menaces rend inquiétantes. Dans les trois cas, enfin, cette crainte des victimes porte sur leur survie elle-même. Dans l'affaire Duvivier, Marie Portal déclare aux policiers qu'elle craignait l'accusé et qu'elle cherchait à l'éviter, car elle savait notamment qu'il " continuait à avoir des idées de vengeance et de mort contre [elle] ${ }^{59}$ ». Dans l'affaire Gonsollin, les craintes de la victime sont exposées dans les très nombreuses pièces $\mathrm{du}$ dossier (plus de deux cent interrogatoires). Anna Lagros y confie à ses camarades qu'elle «craignait beaucoup Gonssolin et [que] c'était par crainte seule qu'elle restait avec lui ${ }^{60}$ ». Les pièces de l'enquête sont unanimes sur les menaces exercées sur elle par son futur agresseur. Une camarade d'Anna Lagros raconte : «Un instant après, je fus rejointe par Gonssolin qui me dit: "Dites à Anna de venir me trouver, autrement il va 
faire vilain ${ }^{61}$ ". » Le commissaire de police qui a entendu la victime confirme les propos de la victime :

Depuis six mois environ ses plaintes devenaient plus fréquentes. Cette fille disait qu'elle avait une grande peur de Gonssolin, qu'elle mourrait un jour et de sa main, [...] et qu'enfin il la frappait sans cesse. Je dois dire que j'avais vu souvent cette fille portant des marques de coups et de meurtrissures. Cette fille me paraissait très effrayée. Je l'engageai à quitter Gonssolin, elle me répondait «si je le quittais, il m'arriverait malheur ». Gonsolin s'arrangeait toujours pour frapper cette fille sans témoin, je ne pouvais pas naturellement donner suite à des plaintes d'une fille soumise, plaintes qu'il ne m'était pas possible de contrôler suffisamment ${ }^{62}$.

Dans l'affaire Grosselin, enfin, la victime était prudente car elle redoutait d'être attaquée par Grosselin, lequel avait été aperçu dans le village qu'elle habitait. Ce sentiment de crainte présent chez les trois victimes avait eu pour conséquence des modifications de leur comportement. Craignant de subir des violences susceptibles de menacer leur vie, elles avaient adopté des attitudes pour tenter de garantir leur survie. Tandis que Marie Descroix « avait redoublé de vigilance ${ }^{63}$ ", Marie Portal avait décidé de quitter l'accusé et de l'éviter, puisqu'il rôdait fréquemment autour de son domicile. Anna Lagros s'était mise, quant à elle, tout d'abord à tenter de cacher les violences subies $^{64}$, puis à échapper à son souteneur ${ }^{65}$. Enfin, Anna Lagros décidait de quitter Gonsollin tout en disant à d'autres, le jour où elle quittait son domicile : "Je ne sais pas ce que j'ai ce soir, je crois qu'il m'arrivera malheur ${ }^{66}$ ! »

Dans ces trois affaires, il apparait en effet que les victimes savent qu'elles sont "tuables», c'est-à-dire qu'elles ont intériorisé qu'elles peuvent être tuées par des hommes, à la fois désignés comme individus (" cet homme ») et comme classe de sexe ( les hommes»). Cette dimension résonne avec la conceptualisation du féminicide chez J. Radford et D.H. Russell, qui identifient le féminicide comme un symptôme du terrorisme sexiste, définissant le terrorisme comme l'imposition d'un régime de terreur visant à maintenir les femmes dans une position subalterne (1992:13-15). Cela est de plus renforcé par le constat de la défaillance de l'institution policière, qui semble incapable de prendre en charge la protection des victimes ou d'agir pour éviter le crime, alors même qu'il apparait à tout le monde que les menaces de mort sont fondées, comme en témoignent les paroles du commissaire de police dans l'affaire Gonssolin. Cette défaillance des institutions censées protéger les femmes pourrait d'ailleurs être intégrée à la définition même des féminicides, tant elle est récurrente au XIX ${ }^{\mathrm{e}}$ comme aujourd'hui ${ }^{67}$, en France comme ailleurs ${ }^{68}$.

De plus, ces dimensions sont à mettre en lien avec un élément prégnant qu'on retrouve dans les trois affaires : la place et le rôle joués par les considérations financières. En effet, il apparait dans les trois affaires étudiées que les hommes qui commettent le crime, bien qu'ils présentent dans leur récit la jalousie comme la motivation première de leurs actions, exploitent leurs victimes et dépendent financièrement d'elles. Dans l'affaire Duvivier, Marie Portal explique avoir quitté l'accusé précisément parce que "c'était un fainéant qui ne travaillait pas depuis dix mois ${ }^{69}$ ", qu'il «ne faisait absolument rien et ne vivait que du produit de mon travail, qui s'élevait à une centaine de francs environ ${ }^{70}$ ", et ce malgré le fait qu'elle « lui avai[t] trouvé une occupation chez M. Mollet, fabricant de cercueil, rue Terne ${ }^{71} »$. Peu après la rupture, Marie Portal rencontre plusieurs fois l'accusé, tombé dans la misère, et lui offre à ces occasions de 
l'argent. Elle remarque qu'il la regarde « de travers ${ }^{72} »$. Dans l'affaire Gonssolin, Anna Lagros accuse son souteneur de lui prendre tout l'argent qu'elle gagne, de la battre si elle ne gagne pas assez. Elle tente même d'expliquer à sa logeuse les raisons pour lesquelles elle ne gagne que peu d'argent: «Voyez Madame Gache, comme il me traite, c'est parce que je ne fais pas assez d'argent. Ce n'est pas de ma faute, je suis malade ${ }^{73}$ ». Dans l'acte d'accusation, on apprend que si Louis Gonssolin avant tant besoin d'argent, c'est du fait de ses addictions au jeu :

[Il était] entièrement dominé par la passion du jeu, ne se livrait à aucun travail et vivait du produit de la prostitution de sa maitresse. Au mois de mars dernier, sa situation était devenue extrêmement critique, ses pertes au jeu avaient tout absorbé, et il se trouvait absolument dénué de ressources ${ }^{74}$. prisme du genre, permet de montrer que l'argent est un révélateur des rapports de pouvoir dans les relations sociales, tout particulièrement lorsque la relation se caractérise par une forte asymétrie. Dans les trois cas en présence, les victimes sont dans une situation financière plus confortable que leurs meurtriers, dont la survie dépend du travail de ces femmes, qu'il s'agisse des revenus de la coiffeuse Marie Portal, de ceux de la prostituée Anna Lagros, ou de la fortune de Marie Descroix, veuve Claitte. L'une des victimes a même pu, à un certain moment, faire don de ses propres ressources à son futur meurtrier. À une époque où le droit permet aux hommes de contrôler les biens de leurs épouses, ces femmes, de par leur statut ou la nature de leur relation à leurs agresseurs, échappent brièvement à la domination économique, habituellement favorable aux hommes. Se trouvant en moins bonne situation matérielle, ceux-ci cherchent à exploiter le travail de ces femmes et à s'accaparer leurs ressources, mais leur stratégie est menacée par le refus qu'elles peuvent, de droit, leur opposer. Bien que les victimes restent dominées au plan social par leurs futurs meurtriers, parce que ceux-ci ont le pouvoir que leur confère leur position d'homme, elles ont, d'un point de vue financier, un modeste pouvoir qui leur permet une capacité de (micro-)résistance, pouvant être assimilée à une forme d'agentivité. En somme, si ces hommes ont le pouvoir de faire craindre aux femmes pour leur intégrité physique, ces femmes peuvent un temps récupérer une capacité d'agir puisque leurs meurtriers dépendent financièrement d'elles.

L'appréhension de cette situation de regain de pouvoir des femmes est permise par l'analyse féministe des archives de féminicides, qui cherche à déceler les logiques de genre à l'œuvre, mais elle ne l'est pas dans la logique de l'enquête judiciaire, dirigée vers la recherche du coupable et du motif du crime. Le seul cas où l'institution policière questionne brièvement cette asymétrie conjoncturelle comme participant à motiver le crime est le cas Grosselin, pour lequel est retenue la qualification d'" homicide motivé par le $v^{7}{ }^{76} »$. Une analyse discursive des propos des hommes meurtriers permet d'ailleurs de saisir les effets que cette brève asymétrie conjoncturelle en faveur des femmes produit chez les meurtriers. Dans l'affaire Duvivier, l'accusé exprime avec amertume et à l'aide de ses propres mots son sentiment d'humiliation : 


\begin{abstract}
Je croyais toujours qu'elle se rappellerait les services que je lui ai rendus [...] car si elle était coiffeuse c'est moi qui ai payé pour lui faire apprendre, et l'argent que j'ai gagné depuis cette époque et celui que j'ai apporté d'autre part, qui m'était dû, celui qu'elle a gagné, devait nous mettre largement dans nos avances, même à l'époque qu'il est, mais l'argent se fondait chez nous, c'est ce qui faisait souvent nos discussions, lorsque je demandai un rendement de compte, ou que je prêchai un peu plus d'économies, bref après m'avoir fait engager tous mes bijoux, et des draps dont j'ai fait vendre une partie depuis, et me trouvant sans travail, elle trouva très logique de me quitter le 3 novembre dernier pour prendre une chambre dans la même maison au $2^{\text {ème }}$, en attendant que je sois parti pour reprendre notre ancienne chambre qu'elle savait bien que je ne garderai pas, vu que le loyer était trop cher pour moi $^{{ }^{77}}$.
\end{abstract}

28 Si le motif présenté par l'institution policière ou le mobile produit par l'auteur pour expliquer son crime sont rarement liés à des considérations financières, hors des cas d'homicide avec vol, ces dimensions, qui s'insèrent dans les rapports sociaux de sexe, apparaissent essentielles pour analyser le féminicide. Elles permettent de mieux saisir la préméditation du crime, caractéristique présente dans les trois affaires et largement établie par les enquêtes, et révèlent que le féminicide est un crime rarement accidentel et très souvent motivé. L'amertume, ou la volonté de se venger d'une situation d'asymétrie favorable à la victime, se nourrissent d'un vif ressentiment qui met en lumière la misogynie des meurtriers. Cet élément, particulièrement perceptible dans le dossier de procédure du cas Duvivier, se manifeste à la fois par la tentative de l'agresseur de tuer par deux fois sa victime ou de lui causer une infirmité ${ }^{78}$ et sous la forme d'un testament laissé à l'attention de la police, qui expose en ces mots sa haine contre sa future victime :

Depuis ce jour j'ai comploté mon projet, j'ai pensé qu'il était utile de débarrasser la société de femme de ce genre-là en rendant la liberté à son homme et je crois que si l'on opérait plusieurs fois dans ces conditions-là, beaucoup de ménage serait plus heureux. Si je dis cela c'est que je n'ai pas eu besoin de la chercher et plus d'une fois elle m'a dit que si ce n'avait pas été moi qui lui tomba sous la main ce serait été un autre, donc elle avait bien envie de commettre sa faute ; je termine mon récit de l'écriture mais je crois qu'à la veille de mourir en plein santé l'on a pas son sang-froid et sa main habituelle ${ }^{79}$.

29 À la lecture de ce document, dont l'authenticité remarquable est attestée par les nombreuses fautes d'orthographe, peu de doutes subsistent quant au ressentiment qui animait l'accusé. Le sentiment d'humiliation perceptible dans ce texte, peut-être causé par les dons d'argent que lui faisait la victime, semble amener l'accusé à passer par le menu les «fautes» (pour reprendre son expression) qu'elle avait commises. Mais il expose ainsi et surtout ce qui l'a amené à agir : la conviction qu'il valait mieux, pour tous les hommes et notamment pour l'ex-mari de la victime, d'éliminer des femmes « de ce genre-là ${ }^{80}$ ». La misogynie du propos de l'accusé est patente dans l'amertume du ton et à travers l'expression "de ce genre-là », qui, si elle reste imprécise, repose sur des représentations stéréotypées et communément admises. On y décèle même une forme de solidarité masculine, puisque l'accusé semble vouloir faire justice à l'ex-mari de la victime par son geste. Cette lecture, peut-être renforcée par l'absence de pluriel (" débarrasser la société de femme de ce genre-là »), même si l'écriture très fautive de Duvivier rend également une simple faute grammaticale probable, rend compte du fait 
que Duvivier n'avait pas accepté la situation de misère dans laquelle il s'était retrouvé à la suite du départ de Marie Portal. Plus que la conduite de cette dernière, il apparait que c'est le sentiment d'humiliation, voire de tromperie ou de moquerie, déclenché par l'inversion, matérielle et symbolique, du lien de dépendance, et par l'utilisation émancipatrice qu'a pu en faire la victime, qui a motivé le passage à l'acte. Enfin, cet écrit forme un témoignage de premier ordre sur la perception consciente des tensions entre la classe des hommes et celles des femmes : il y aurait, en effet, une légitimité des hommes, pour le bien de « la société », à tuer des femmes jugées immorales.

\section{Conclusion}

Le présent article a cherché à rendre compte de l'apport de l'analyse féministe dans l'étude des cas d'archives de féminicides, poursuivant ainsi les travaux amorcés par les chercheuses féministes, à l'instar d'Arlette Farge. Étudier en féministe les féminicides dans les archives judiciaires requiert tout d'abord des stratégies de contournement des catégories universelles du droit pour définir un matériau d'enquête à soi. S'essayer à analyser ces archives au prisme du genre permet ensuite d'identifier les logiques et les pratiques lors de l'enquête judiciaire et policière, qu'elles soient le fait des hommes accusés du crime ou des acteurs de la procédure. La grille de lecture adoptée par les acteurs procède de stéréotypes genrés qui, tout en mobilisant des catégories universelles du droit, font précisément obstacle à une appréhension genrée du crime qui soit attentive aux rapports sociaux de sexe lors des féminicides. Ainsi, alors même qu'elles nourrissent les dynamiques de genre dans le traitement institutionnel des féminicides, les pratiques judiciaires ne permettent pas l'émergence de la catégorie unitaire d'un féminicide qui soit sous-tendue par la question du genre. Au contact de ces cas, une perspective féministe, qui tente de rompre avec la lecture conjugaliste des féminicides tout en reconnaissant leur importance dans la lutte pour la reconnaissance des violences faites aux femmes, s'avère utile pour montrer qu'au-delà des catégories hétérogènes du droit, la lecture sociale des données fait émerger des traits récurrents : rôle central de la question financière, sentiment d'humiliation des hommes lié à un amoindrissement conjoncturel de leur domination sur les femmes, tentative d'émancipation des victimes. Des meurtres de femmes a priori hétérogènes trouvent alors leur unité sous la catégorie féministe de féminicide, laquelle participe à mieux saisir les logiques pour lesquelles les hommes font violence aux femmes.

\section{BIBLIOGRAPHIE}

BERGER, Virginie. 2004. «Les plans de l'enquête dans la seconde moitié du XIX ${ }^{\mathrm{e}}$ siècle », Sociétés Représentations 18 (2) : 97-107.

BODIOU, Lydie, CHAUVAUD, Frédéric, GAUSSOT, Ludovic, GRIHOM, Marie-José \& LAUFER Laurie (éds.). 2019. On tue une femme : le féminicide, histoire et actualités. Paris : Hermann. 
CACOUAULT-BITAUD, Marlaine \& JASPARD, Maryse. 2020. « Féminicide : une qualification nécessaire... et suffisante? » Travail, genre et sociétés 43 (1) : 145-147.

CHOULET-VALLET, Anaïs, CLOCHEC, Pauline, FRASCH, Delphine, GIACINTI, Margot \& VÉDIE Léa. 2021. Théoriser en féministe. Paris : Hermann.

CLAIR, Isabelle. 2016. « Faire du terrain en féministe » Actes de la recherche en sciences sociales 213 (3) : 66-83.

CLAVERIE, Élisabeth. 1979. « L'honneur » : une société de défis au XIX ${ }^{\mathrm{e}}$ siècle » Annales 34 (4) : 744-759.

CORBIN, Alain. 1978. Les Filles de noce. Misère sexuelle et prostitution aux XIXe et XX'e siècles. Paris : Aubier.

CRÉPIN, Marie-Yvonne. 2016. «L'homicide du conjoint en Bretagne aux XVIII ${ }^{\mathrm{e}}$ et XIX ${ }^{\mathrm{e}}$ siècles : permanence d'un crime familial » Annales de démographie historique 130 (2) : 51-68.

DEBAUCHE, Alice, HAMEL, Christelle \& KAC-VERGNE, Marianne. 2013. « La violence comme contrôle social des femmes » Nouvelles Questions Féministes 32 (1) : 96-111.

DELAGE, Pauline. 2017. Violences conjugales : du combat féministe à la cause publique. Paris : Presses de Sciences Po.

DEVINEAU, Julie. 2012. « Autour du concept de fémicide/féminicide : entretiens avec Marcela Lagarde et Montserrat Sagot » Problèmes d'Amérique latine 84 (2) : 77-91.

FALQUET, Jules. 2016. Pax neoliberalia. Paris : Éditions iXe.

FARCY, Jean Claude, KALIFA, Dominique \& LUC, Jean-Noël (éds.). 2007. L'enquête judiciaire en Europe au XIX ${ }^{e}$ siècle : acteurs imaginaires pratiques. Grâne : CREAPHIS.

FARCY, Jean-Claude. 2001. « La gendarmerie, police judiciaire au XIX ${ }^{\mathrm{e}}$ siècle » Histoire, économie \& société 20 (3) : 385-403.

FARGE, Arlette. 1997 [1981]. Le goût de l'archive. Paris : Éditions du Seuil.

FERRON, Laurent. 2015. « Le témoignage des femmes victimes de viols au XIX siècle », in Femmes et justice pénale : XIX ${ }^{e}-X X^{e}$ siècles, BARD, Christine, CHAUVAUD, Frédéric, PERROT, Michelle \& PETIT, Jacques-Guy (éds.). Rennes : Presses universitaires de Rennes, 29-138.

FOUGEYROLLAS-SCHWEBEL, Dominique \& JASPARD, Maryse. 2003. « Compter les violences envers les femmes. Contexte institutionnel et théorique de l'enquête ENVEFF »Cahiers du Genre 35 (2) : 45-70.

GAGNON, Gemma. 2002. «L'homicide conjugal et la justice française au XIX siècle », in Femmes et justice pénale : XIX ${ }^{e}-X X^{e}$ siècles, BARD Christine, CHAUVAUD Frédéric, PERROT Michelle \& PETIT Jacques-Guy (éds.). Rennes : Presses universitaires de Rennes, 139-147.

GIACINTI, Margot. 2020. «"Nous sommes le cri de celles qui n'en ont plus“ : historiciser et penser le féminicide » Nouvelles Questions Féministes 39 (1) : 50-65.

GUILHAUMOU, Jacques. 2006. Discours et événement : l'histoire langagiere des concepts. Besançon : Presses universitaires de Franche-Comté.

HANMER, Jalna. 1977. « Violence et contrôle social des femmes » Questions Féministes $1:$ 68-88.

HOUTE, Arnaud. 2009. « La fabrique du procès-verbal dans la France du XIX ${ }^{\mathrm{e}}$ siècle : contribution à l'histoire de l'écrit administratif » Atelier du Centre de recherches historiques 5. 
ISRAËL, Liora. 2012. «L'usage des archives en sociologie », in L'enquête sociologique, PAUGAM Serge (éd.). Paris » Presses Universitaires de France, 167-185.

KALIFA, Dominique. 2010. « Enquête et "culture de l'enquête“ au XIX"e siècle » Romantisme 149 (3) : 3-23.

LAZAAR, Fiona. 2020. Rapport d'information n²695 sur la reconnaissance du terme de " féminicide ». Paris : Assemblée Nationale.

MELCARE-ZACHARA, Johanne. À paraitre. « L'apparition des circonstances atténuantes en matière criminelle » Cahiers historiques des Annales de droit.

NIEDERGANG, Pierre \& PITERBRAUT-MERX, Tal. 2021. « Violence sexuelle ou "initiation“? » GLAD! [En ligne], 10, consulté le 08 novembre 2021. URL : https://journals.openedition.org/glad/ 2734.

NUGARA, Silvia. 2011. L'émergence de violence domestique comme rubrique du discours institutionnel : le cas du Conseil de l'Europe. Thèse de doctorat en science du langage. Paris : Université Paris 3.

PITERBRAUT-MERX, Tal. 2020. « Enfance et vulnérabilité. Ce que la politisation de l'enfance fait au concept de vulnérabilité » Éducation et socialisation [En ligne], 57, consulté le 08 novembre 2021. URL : https://journals.openedition.org/edso/12317.

PLUMAUZILLE, Clyde. 2016. Prostitution et révolution: les femmes publiques dans la cité républicaine (1789-1804). Ceyzérieu: Champ Vallon.

RUSSELL, Diana. 2001. "Defining femicide and related concepts”, in Femicide in global perspective, RUSSELL Diana \& HARMES Roberta (éds.). New York: Teacher's College Press, 12-28.

RADFORD, Jill \& RUSSELL, Diana (éds.). 1992. Femicide: the politics of woman killing. New York: Twayne Publishers Inc.

RONAI, Ernestine \& DURAND Édouard (éds.). 2017. Violences conjugales : le droit d'être protégée. Paris : Dunod.

SICARD, Germain. 1993. «Doctrine pénale et débats parlementaires : la réformation du Code pénal en 1831-1832 » Revue d'histoire des Facultés de droit et de la science juridique 14 : 137-165.

SEGATO, Rita Laura. 2016. La guerra contra las mujeres. Madrid: Traficantes de Sueños.

TANGUY, Jean-François. 2015. « Les victimes de violences conjugales en Bretagne au XIX ${ }^{\mathrm{e}}$ siècle », in Les victimes, des oubliées de l'histoire?, GARNOT Benoît (éd.). Rennes : Presses universitaires de Rennes, 259-277.

TATOUIEX, Laura. 2018. L'avortement en France à l'époque moderne. Entre normes et pratiques (mi $X V I^{e}$-1791). Thèse de doctorat en histoire. Rouen : Normandie Université.

TILLIER, Annick. 2001. Des criminelles au village : femmes infanticides en Bretagne, 1825-1865. Rennes : Presses universitaires de Rennes.

VANNEAU, Victoria. 2016. La paix des ménages : histoire des violences conjugales, XIX ${ }^{e}-X X I^{e}$ siècle. Paris : Anamosa.

VERJUS, Anne. 2010. Le bon mari : une histoire politique des hommes et des femmes à l'époque révolutionnaire. Paris : Fayard. 


\section{ANNEXES}

\section{Sources archivistiques (par ordre chronologique) :}

AD69, 2U299 Affaire Pidal (1869).

AD69, 2U365 Affaire Talagrand (1876).

AD69, 2U380 Affaire Duvivier (1878).

AD69, 2U387 Affaire Vermorel (1878).

AD69, 2U402 Affaire Laurent (1880).

AD69, 2U411 Affaire Gonssolin (1880).

AD69, 2U414 Affaire Mercier (1880).

AD69, 2U419 Affaire Hervier (1881).

AD69, 2U445 Affaire Bonnin (1883).

AD69, 2U503 Affaire Grosselin (1888).

Sources législatives (par ordre chronologique) :

Code civil de 1804.

Code d'instruction criminelle de 1808.

Code pénal de 1810, articles 2, 295, 296, 299, 300, 317.

Loi du 25 juin 1824.

Loi de 1832, article 309.

Loi du 21 novembre 1901.

Loi du 17 janvier 2017, article 132-77.

\section{NOTES}

1. Je fais ici référence par exemple à la page Facebook « Féminicides par ex ou par compagnon », essentielle dans la constitution de la mobilisation contre les féminicides, qui a représenté dès 2014 l'une des premières initiatives militantes de comptage des féminicides. Une partie des militantes engagées dans cette dynamique se sont rassemblées en association en 2019 sous le nom « Union des Familles de Féminicides ». Voir leur site internet : https://unff.fr/ (consulté le 20/09/2021).

2. On peut citer par exemple le Grenelle des violences conjugales, initiative gouvernementale mise en place à partir du 9 septembre 2019, notamment par les services de la secrétaire d'État chargée de l'Égalité entre les femmes et les hommes et de la Lutte contre les discriminations de l'époque, Marlène Schiappa. Les objectifs institutionnels du Grenelle sont visibles sur le site du ministère : https://www.egalite-femmes-hommes.gouv.fr/3-9-19-grenelle-des-violencesconjugales/ (consulté le 20/09/2021).

3. Cette définition est actuellement acceptée autant sur le plan social que légal, comme en témoignent les récents débats au sujet de l'incorporation du terme de féminicide dans la législation pénale française. À titre d'exemple, le rapport d'information de l'Assemblée Nationale en date du 18 février 2020 visant à étudier la reconnaissance du terme de féminicide propose une réflexion sur cette intégration légale à partir de cas de féminicides conjugaux. Ce rapport met en 
évidence les dangers que représenterait la création d'une infraction autonome de féminicide pour l'égalité des citoyen'ne's devant la loi et pour l'universalisme du droit, mais exhorte dans le même temps à utiliser le terme dans les dispositifs institutionnels, en particulier dans « toutes les formations sur les violences conjugales» (Lazaar 2020: 25), ce qui semble paradoxalement reconnaitre au concept son caractère opératoire pour appréhender ce fait social.

4. Ce constat est spécifique à la France et présente depuis le milieu de l'année 2021 quelques évolutions. En effet, certains comptages, comme celui réalisé par le collectif «Féminicides par compagnons ou ex", recensent de plus en plus les féminicides hors de la seule sphère conjugale. Par ailleurs, la littérature portant sur l'étude des féminicides en Amérique Latine conçoit de manière plus large le féminicide (Falquet 2016).

5. Pour des exemples concrets, voir le numéro "Droit et Féminisme : quelles expertises?", Chronique Féministe, janvier-juin 2018. On peut toutefois citer des évolutions récentes, qui visent à faire sortir le droit de cette perspective conjugaliste et familialiste, comme par exemple la modification de l'article 132-77 du code pénal par la loi du 17 janvier 2017, qui aggrave la peine encourue si «le crime ou le délit est précédé, accompagné ou suivi de propos, écrits, images, objets ou actes de toute nature qui soit portent atteinte à l'honneur ou à la considération de la victime ou d'un groupe de personnes dont fait partie la victime à raison de son sexe, son orientation sexuelle ou identité de genre vraie ou supposée, soit établissent que les faits ont été commis contre la victime pour l'une de ces raisons" (En ligne, consulté le 20/09/2021. URL : https://www.legifrance.gouv.fr/jorf/article_jo/JORFARTI000033935146. Nous soulignons).

6. Cette lecture se couple souvent à une lecture familialiste, comme en témoigne le «protocole féminicide » instauré sur recommandation de l'observatoire des Violences envers les Femmes de Seine-Saint-Denis et du Parquet du tribunal de Bobigny, qui vise à protéger les enfants covictimes dans le cas du féminicide d'un·e de leurs proches parent·es, généralement leur mère (Ronai \& Durand 2017). Si l'on peut à la fois souligner l'importance d'une telle prise en charge et regretter l'absence de la généralisation d'un tel dispositif à l'ensemble du territoire français, l'appellation choisie ("protocole féminicide»), considérant le public ciblé - les enfants interroge. En effet, l'appréhension d'un tel dispositif suggère qu'il vise à protéger les femmes, alors même qu'il intervient après que les femmes aient été assassinées. Il paraitrait d'ailleurs intéressant d'interroger davantage la catégorie de «co-victimes » dans le cadre des féminicides, en particulier lorsqu'elle suggère que le féminicide est aussi une violence faite aux enfants. Sur ces dernières, nous renvoyons aux travaux éclairants de Tal Piterbraut-Merx, doctorant et amie qui nous a quittée's récemment (Piterbraut-Merx 2020 ; Niedergang \& Piterbraut-Merx 2021).

7. Nous faisons ici référence à la démarche d'enquête féministe telle que notamment défendue dans plusieurs ouvrages récents (voir Clair, 2016 ou Choulet-Vallet et al., 2021).

8. Cette posture, qui vise à saisir la dynamique de genre dans les archives, peut s'apparenter à ce que Jacques Guilhaumou appelle «le geste de lecture» qu'implique l'usage de l'archive par l'historien'ne, c'est-à-dire non seulement le fait de situer l'énonciation dans l'histoire, mais aussi de lire l'évènement à l'aune d'une temporalité contemporaine et d'interroger son énonciation par rapport aux discours dominants (Guilhaumou 2006).

9. Cet article s'inscrit dans le cadre d'une recherche doctorale qui s'intéresse aux féminicides de 1791 à nos jours. Je choisis ici de me concentrer plus particulièrement sur le XIX ${ }^{\mathrm{e}}$ siècle, car se figent à cette époque, avec le Code pénal de 1810, des catégories pénales qui resteront pratiquement inchangées jusqu'en 1994. De plus, les règles de communicabilité permettent de consulter l'ensemble des sources judiciaires sans limitation, contrairement aux dossiers de procédures pénales de moins de 75 ans (voire de 100 ans si la victime était mineure au moment des faits) pour lesquels il faut demander une dérogation.

10. Compte tenu de la définition utilisée du féminicide, le genre de l'accusée n'est pas déterminant dans la sélection des dossiers, puisque le féminicide n'est pas déterminé par le genre de l'accusée, mais par le genre de la victime. Toutefois pour cette étude, nous nous limiterons 
aux cas d'hommes ayant tué des femmes, ce qui explique le choix de ne pas écrire en écriture inclusive "auteur du crime", "accusé ", etc. Outre les différences dans les féminicides dans lesquels le meurtrier est un homme et les féminicides dans lesquels la meurtrière est une femme, l'écrasante majorité des dossiers de féminicides sont le fait d'hommes sur des femmes, d'où notre choix de traiter ce type de féminicides. De plus, on peut noter que tous les dossiers présentant des cas d'homicides dont la victime est une femme ne sont pas nécessairement des féminicides.

11. L'assassinat est défini par l'article $296 \mathrm{du}$ Code pénal de 1810 comme "l'homicide prémédité ", tandis que le meurtre est défini comme «l'homicide non prémédité " par l'article $295 \mathrm{du}$ Code pénal.

12. Le parricide est défini par l'article 299 du Code pénal de 1810 comme « le meurtre des pères ou mères légitimes, naturels ou adoptifs, ou de tout autre ascendant légitime ».

13. L'article 300 définit l'infanticide comme «le meurtre de l'enfant nouveau-né ». Cette catégorie sera modifiée par la loi du 21 novembre 1901 qui fait de l'infanticide «le meurtre ou l'assassinat de l'enfant nouveau-né ».

14. L'article 301 définit l'empoisonnement comme "tout attentat à la vie d'une personne, par l'effet de substances qui peuvent donner la mort plus ou moins promptement, de quelque manière que ces substances aient été employées ou administrées, et quelles qu'en aient été les suites ».

15. L'article 2 du Code pénal de 1810 considère que «toute tentative de crime qui aura été manifestée par des actes extérieurs, et suivie d'un commencement d'exécution, si elle n'a été suspendue ou n'a manqué son effet que par des circonstances fortuites ou indépendantes de la volonté de l'auteur, est considérée comme le crime même ».

16. Toutefois, il arrive que la victime décède au cours de l'information judiciaire sans pour autant que le crime soit requalifié en meurtre ou en assassinat. L'accusé peut donc être condamné pour tentative de meurtre, alors que la victime est décédée.

17. La mort occasionnée à la suite de coups ou de blessures n'était pas envisagée dans le Code pénal de 1810. L'article 309 était ainsi fait : «Sera puni de la peine de la réclusion, tout individu qui aura fait des blessures ou porté des coups, s'il est résulté de ces actes de violence une maladie ou incapacité de travail personnel pendant plus de vingt jours ».

18. Voir à ce sujet Sicard 1993.

19. L'article 309 modifié par la loi de 1832 dispose que «Si les coups portés ou les blessures faites volontairement, mais sans intention de donner la mort, l'ont pourtant occasionnée, le coupable sera puni de la peine des travaux forcés à temps. ».

20. Si l'avortement est défini par l'article 317 du Code pénal de 1810 et ainsi différencié de l'infanticide, les récits des femmes accusées d'infanticide permettent de rendre compte que la logique de l'infanticide en période d'interdiction de l'avortement est dans certains cas assimilable à la logique de l'avortement. Lire à ce sujet la thèse de Laura Tatoueix (2018), qui donne matière à réflexion au sujet de l'avortement du XVI ${ }^{\mathrm{e}}$ siècle à la Révolution française.

21. Pour plus d'informations sur l'enquête policière au XIX ${ }^{\mathrm{e}}$ siècle, voir Berger 2004, Farcy 2001, Farcy, Kalifa \& Luc 2007, Houte 2009 et Sicard 1993.

22. Dans l'affaire Pidal (1869, AD69, 2U299), l'acte d'accusation indique que «ses aveux ont été complets. Il n'a caché ni les motifs divers qui l'avaient poussé au meurtre, si sa longue préméditation et depuis il a constamment persisté dans ses déclarations dont la sincérité a été constatée. »

23. Le corpus étudié est celui de ma thèse de doctorat de science politique en cours au Laboratoire Triangle (UMR 5206), ENS de Lyon, provisoirement intitulée : « Le féminicide : enjeux socio-historiques d'une catégorisation juridique dans l'espace francophone, de la Révolution française à nos jours ".

24. AD69, 2U402 Affaire Laurent.

25. AD69, 2U380 Affaire Duvivier. 
26. AD69, 2U365 Affaire Talagrand.

27. AD69, 2U445 Affaire Bonnin.

28. Les circonstances atténuantes, qui permettent d'amoindrir les peines, sont introduites par la loi du 25 juin 1824.

29. AD69, 2U419 Affaire Hervier, nous soulignons.

30. Ibid.

31. AD69, 2U387 Affaire Vermorel.

32. Ibid.

33. Le terme «fille soumise » renvoie à la catégorie utilisée dans les archives pour désigner les femmes exerçant la prostitution.

34. AD69, 2U414 Affaire Mercier.

35. Ibid.

36. AD69, 2U414 Affaire Mercier.

37. Ibid.

38. Ibid.

39. Ibid.

40. Ibid.

41. Ibid.

42. Ibid.

43. La condamnation de Mercier est de six mois de prison et de 100 francs d'amende. Sur les 20 féminicides où des hommes ont tué des femmes entre 1876 et 1886 dans le Rhône (21 accusés), deux sont condamnés à mort, cinq sont condamnés aux travaux forcés à perpétuité, cinq sont condamnés à 10 ans ou plus de travaux forcés, un est condamné à moins de 10 ans de travaux forcés, 4 sont condamnés à plus d'un an et jusqu'à 10 ans de prison, 3 sont condamnés à 1 an ou moins de prison, et 1 (complice d'un crime) est acquitté (données personnelles).

44. AD69, 2U380 Affaire Duvivier, nous soulignons.

45. Ibid.

46. AD69, 2U387 Affaire Vermorel, nous soulignons.

47. La relation entre l'agentivité des femmes face aux hommes et le processus conduisant au féminicide doit faire l'objet d'une étude approfondie, que nous réservons pour un travail à paraitre prochainement.

48. AD69, 2U380 Affaire Duvivier.

49. Ibid.

50. AD69, 2U380 Affaire Duvivier.

51. AD69, 2U411 Affaire Gonssolin.

52. Ibid.

53. Ibid.

54. AD69, 2U503 Affaire Grosselin.

55. AD69, 2U503 Affaire Grosselin.

56. Ibid.

57. Ces dénominations ne sont pas des dénominations officielles, nous nous permettons ici de résumer ce qui est mis en avant dans les dossiers.

58. La citation d'A. Farge concerne le XVIII ${ }^{e}$, mais elle se vérifie également au XIX ${ }^{\mathrm{e}}$ siècle.

59. AD69, 2U380 Affaire Duvivier.

60. AD69, 2U411 Affaire Gonssolin.

61. AD69, 2U411 Affaire Gonssolin

62. Ibid.

63. AD66, 2U503 Affaire Grosselin. 
64. Ceci est notamment prouvé par un témoignage : «Elle a porté pendant plusieurs jours aussi un foulard autour du cou pour cacher les traces de violences faites sur son cou.» (AD69, 2U411 Affaire Gonssolin).

65. Dans une audition, une témoin indique : «Elle me dit : "Je vais me sauver voilà mon homme, il va me battre, je n'ai rien fait ce soir”. Elle était effrayée, elle me donna son parapluie et se sauva dans la rue Saint Joseph où j'allais la rejoindre. » (AD69, 2U411 Affaire Gonssolin).

66. AD69, 2U411 Affaire Gonssolin.

67. Au 9 novembre 2021, selon les chiffres du collectif « Féminicides par ex ou par compagnon », qui recense les féminicides et les tiers victimes, 99 femmes ont été tuées, alors même que certaines d'entre elles étaient prises en charge parce qu'elles étaient victimes de violence conjugales et que certains auteurs étaient déjà connus des services de police.

68. Cette dimension rappelle d'ailleurs l'ajout proposé par l'anthropologue Marcela Lagarde de l'impunité de l'État et de la défaillance des institutions dans la protection des victimes dans la définition originelle du féminicide (femicide) de Radford et Russell (Devineau 2012).

69. AD69, 2U380 Affaire Duvivier.

70. Ibid.

71. AD69, 2U380 Affaire Duvivier.

72. Ibid.

73. AD69, 2U411 Affaire Gonssolin. Pour plus de détails sur la situation des prostituées au XIX voir Corbin 1978.

74. Ibid.

75. AD66, 2U503 Affaire Grosselin.

76. Ibid.

77. AD69, 2U380 Affaire Duvivier.

78. Ibid. Dans le message laissé par Duvivier, on peut lire : «Voilà quinze jours que j'attends l'occasion [...] de pouvoir la ressaisir pour qu'elle ait au moins un souvenir de moi si je ne la tue pas. »

79. Ibid. Texte reproduit avec ses fautes de langue.

80. Idem.

\section{RÉSUMÉS}

Cet article s'intéresse aux affaires de féminicides dans les archives judiciaires du XIX ${ }^{\mathrm{e}}$ siècle. Si le féminicide, dans son acception contemporaine en France, est souvent réduit à sa dimension conjugale (meurtre d'une épouse par son époux), l'étude des archives de féminicides à partir de la définition donnée par Radford et Russell (1992) permet de rendre compte d'une diversité de cas de femmes tuées par des hommes et d'identifier des logiques de genre déterminantes dans le crime. L'article s'interroge alors sur ce qui a pu faire écran à une telle lecture, en s'intéressant aux pratiques des acteurs lors de l'enquête judiciaire, puis en étudiant trois affaires a priori très différentes dans lesquelles la victime est une femme. L'article conclut à l'existence de traits récurrents qui poussent les hommes à tuer les femmes, qui attestent de l'exercice de la domination masculine, et qui permettent de subsumer les cas présentés sous la catégorie unitaire de féminicide. 
This article focuses on cases of femicides in $19^{\text {th }}$ century court records. Although femicide, in its contemporary understanding in France, seems to be reduced to its marital dimension (murder of a wife by her husband), the study of femicide archives based on Radford and Russell's definition (1992) makes it possible to account for the diversity of cases of women killed by men and to identify the determining gender factors in the crime. Thus, the paper examines what might have prevented such a reading by looking at the practices of the actors involved in the judicial investigation, and by studying three - at first sight very different - cases in which the victim is a woman. The article concludes that there are recurrent factors that lead men to kill women, which demonstrate the exercise of male domination, and which allow the cases presented to be subsumed under the unitary category of femicide.

\section{INDEX}

Thèmes : Recherches

Keywords : femicide, archives, 19th century, France, socio-history

Mots-clés : féminicide, archives, XIXe siècle, France, sociohistoire

\section{AUTEUR}

\section{MARGOT GIACINTI}

Margot Giacinti est doctorante en science politique à l'ENS de Lyon (UMR 5206, Laboratoire Triangle) et ATER en science politique à l'Université Lyon 2 Lumière. Elle réalise une thèse sur le féminicide et les enjeux socio-historiques de sa catégorisation juridique en France, de la Révolution française à nos jours, sous la direction d'Anne Verjus. 